\title{
Laser Scribing on Silver Flexible Substrate with Beam Shaping Technology
}

\author{
Ho-Chiao Chuang* and You-Chang Lee \\ Department of Mechanical Engineering, National Taipei University of Technology \\ No. 1, Sec. 3, Chung-Hsiao East Rd., Taipei 10608, Taiwan \\ E-mail: hchuang@mail.ntut.edu.tw
}

\begin{abstract}
It is well-known that the use of laser beam with Top-hat profile is suitable for selective ablation of thin films. The main challenge to develop such applications is the beam shaping step to transform a high coherent (low $\mathrm{M}^{2}$ ) Gaussian beam in a perfect Top-hat profile. Thus, many studies on laser beam shaping technology are carried out recently. In this study, the material for laser scribing experiments is an Ag / PET (Polyethylene Terephthalate) thin film. A U-shaped cross-sectional profile of the $\mathrm{Ag} / \mathrm{PET}$ film is generated by the laser scribing process with a wavelength of $532 \mathrm{~nm}$ nanosecond laser system. However, a V-shaped cross-sectional profile of the Ag / PET thin film is generated with a picosecond laser system using the same wavelength. The shape of the scribed line is mainly related to the beam profile for the pulse durations investigated. In order to produce the same Ushaped cross-sectional profile with a picosecond laser system $(532 \mathrm{~nm})$, a beam shaper was used in this study to obtain a Top-hat beam for laser scribing experiments. A laser beam profiler is used to examine the laser beam shape and a metallurgical microscope is used to observe the cross-sectional profile of the scribed samples. After laser scribing experiments, the cross-sectional profiles of samples are also recorded by a Laser Scanning Confocal Microscopy (LSCM). In conclusion, a Ushaped cross-sectional profile is successfully obtained by using a picosecond laser with a beam shaper that significantly reduces the damage on the substrate, PET thin film.
\end{abstract}

DOI: $10.2961 / \mathrm{jlmn} .2014 .03 .0003$

Keywords: beam shaping, Top-hat beam, picosecond laser, Ag/PET thin film

\section{Introduction}

$\mathrm{Ag} / \mathrm{PET}$ film is mainly used in the touch panel industry, and the main purpose is to connect ITO (Indium thin oxide) and the touch IC. The traditional manufacturing method is screen printing $[1,2]$. However, the finer line patterns cannot be produced by the screen printing process, and therefore additional processes are needed. The current solution is to print out all the line patterns by the screen printing first including the line patterns which needs to be removed later. The unwanted conductive silver adhesives are then removed by the post processes. Currently, after the screen printing process, $\mathrm{Ag} / \mathrm{PET}$ thin films need to go through the traditional wet etching process which creates the environmental pollution. However, if the laser scribing process can be implemented for the $\mathrm{Ag} / \mathrm{PET}$ thin films removing process, it can not only reduce the pollution of the environment but also to reduce the cost. Therefore, laser scribing has a great advantage on the $\mathrm{Ag} / \mathrm{PET}$ film lines patterning process and thus becomes an important developing industry.

Venkat et al [3] reports the laser scribing processes on the ITO film uses the methods of direct writing, mask projection and three different laser sources. The crystals of the three different laser sources are $\mathrm{Nd}: \mathrm{YVO}_{4}, \mathrm{Nd}$ : YLF and $\mathrm{Nd}$ : YAG. From their test results, Nd: $\mathrm{YVO}_{4}$ laser has the highest pulse repetition rate. Thus, in this study an $\mathrm{Nd}$ : $\mathrm{YVO}_{4}$ laser is selected as the laser scribing source, since a poor laser pulse repetition rate may adversely affect the quality of laser scribed line patterns which results in varying on the width and depth of line patterns, causing the disconnection on Ag/PET thin film. Raciukaitis [4] used a high repetition rate picosecond laser at different wave- lengths (266, 355 and 532nm) to perform the scribing process on an ITO thin film. The scribed line patterns are examined by the SEM and AFM, and the amount of laser energy needed to remove the ITO film was also analyzed. Different laser wavelengths requires different energy in the laser scribing process parameters. In their study, different laser wavelengths and fluence were used such as $266 \mathrm{~nm}$ $\left(0.2 \mathrm{~J} / \mathrm{cm}^{2}\right), 355 \mathrm{~nm}\left(0.46 \mathrm{~J} / \mathrm{cm}^{2}\right)$ and $532 \mathrm{~nm}\left(1.55 \mathrm{~J} / \mathrm{cm}^{2}\right)$. From their results, it shows that the best quality of a scribed groove was obtained by using a laser wavelength of $355 \mathrm{~nm}$, but there will be a lot of dust residue on the sample surface. However, the best surface quality after scribing (no dust) was observed by using a laser wavelength of $266 \mathrm{~nm}$. Therefore, UV picosecond lasers with a high repetition rate were found to be a good alternative to the conventional photolithography for flexible patterning of ITO. Park et al [5] used an ultrafast laser $(810 \mathrm{~nm})$ with a pulse width of 150 femtosecond, a mode-locked Ti: sapphire oscillator and a regenerative amplifier to perform the scribing process on an OLED film of an ITO/glass substrate. Their results show that the resistance value of the ITO is significantly affected by the scribed surface quality and the shape of grooves according to the above laser scribing conditions. The glass substrate was found undamaged when the laser energy density was set to $0.07 \mathrm{~J} / \mathrm{cm}^{2}$ and the substrate was selectively damaged when the laser energy density was set to about 1.2 $\sim 1.6 \mathrm{~J} / \mathrm{cm}^{2}$. Their experiments proved that an ultrafast laser scribing process can produce a high aspect ratio pattern on the ITO layer and without making any damages on the glass substrate. 
The profile of a typical laser beam is the Gaussian distribution and the center of the laser intensity is the strongest part which has significant drawbacks during the laser scribing process. For example, from our scribing results, it shows that a deeper depth is created in the middle part of a workpiece which damages the PET substrate due to the Gaussian distribution of the laser beam instead of a Top-hat distribution. Thus, more studies are focused on the topic of the laser beam shaping [6]. In addition, a laser scribing process with a general Gaussian beam also causes the uneven heating of the entire workpiece surface which leads to form a V-shaped cross-sectional profile of the Ag/PET thin film. A V-shaped cross-sectional profile has a smaller linewidth and a weaker material strength of the substrate compared to a U-shaped cross-section profile. Moreover, the bottom substrate material of a V-shaped profile, PET, has a deeper scribed groove that makes the strength of the substrate become weaker or even damages the substrate as shown in Fig. 1. Therefore, the wet etching process cannot be replaced by the laser scribing process.

Recently, many studies have shown that the Top-hat laser beam is superior than the Gaussian beams on laser processing, for example the laser annealing technology is used on ITO thin film [7]. It shows that the conductivity and surface roughness of the ITO surface are improved after laser annealing processes. In addition, the curing time of the metallic ink can be substantially reduced with the assistance of the laser shinning which not only to improve the yield of metallic ink printing but also to increase the conductivity and the hardness of the printed metallic ink [8]. Furthermore, Chen et al used a third harmonic Nd:YAG laser processing system with the laser beam shaping technology for removing the ITO thin films on glass substrate [6], and their results show that the original Gaussian beam profile can be converted into a Top-hat beam profile with a beam shaper. The scribed line patterns are uniform, smooth and no damages were found on the glass substrate.

In this study, a picosecond laser system with a wavelength of $532 \mathrm{~nm}$ is used to perform the laser scribing experiments on $\mathrm{Ag} / \mathrm{PET}$ thin film with different kinds of beam shaping profiles. The results prove that with beam shaping technology, the damage of the substrate Ag/PET can be improved after the scribing process and thus enhance the strength of the substrate PET and silver layer as shown in Fig. 1.

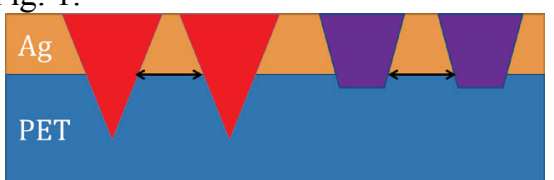

Fig. 1 Schematic diagram of the U-shaped and V-shaped cross-sectional profiles. Under the same line width, the PET substrate is less damaged with the U-shaped groove, compared to the V-shaped groove.

\section{Picosecond laser processing system}

This study used an $\mathrm{Nd}^{-} \mathrm{YVO}_{4}$ (Neodymium: Yttrium Orthovanadate) picosecond laser with a wavelength $532 \mathrm{~nm}$ and a maximum output power $14 \mathrm{~W}$. The laser optical system is shown in Fig. 2, the laser beam goes through a beam splitter first and divides into two beams. The first beam penetrates the beam splitter for the scribing process and the other beam is for the purpose of power detection. The first laser beam is expanded after passing through a beam expander and then goes into a beam shaper. After the beam shaper, it is focused on the Ag/PET films by adjusting a focusing lens for scribing process. The focusing lens is mounted on the Z-axis and the laser scribing process is implemented by moving the XY stage which is the laser direct writing. The main purpose of this study is not on optimizing the laser scribing parameters; thus in this study the laser beam shaping profiles and the cross-sectional shape of the workpiece are explored only under a fixed laser processing parameters at frequency $(\mathrm{kHz})$, power, focus $(\mathrm{mm})$ and the laser pulse width.

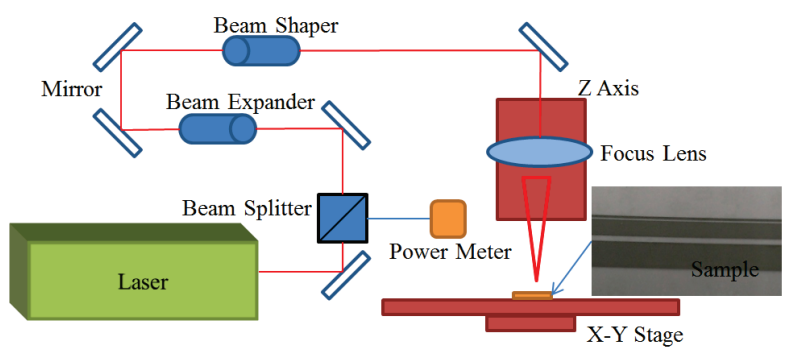

Fig. 2 Schematic diagram of optical setup for the laser scribing system.

\section{Beam shaping technology}

Due to inhomogeneous laser intensity distribution (Gauss beam), the amount of unused laser energy could reach a very high level. Therefore, with the use of beam shaping technology, it would help to save the laser energy, which is very important for modern laser technology, especially in materials processing [9]. The definition of laser beam shaping is a process to reallocate the light field and its phases of the radiated light rays [10]. The distribution of the optical field defines the beam shape, such as Gaussian beam or Top-hat beam. Typical laser beam shaping can be divided into three kinds [11]: aperturing, field mapping, and multiaperture beam integrator. Gaussian beams can be converted into Top-hat beams by any of the above methods. The beam shaper (Focal- $\pi$ shaper 9_532, AdlOptica GmbH, Berlin, Germany) used in this study is to convert the laser Gaussian distribution into an airy disk distribution first [12] and then change to the Top-hat beam via a focusing lens for the purpose of laser scribing process as shown in Fig. 3.

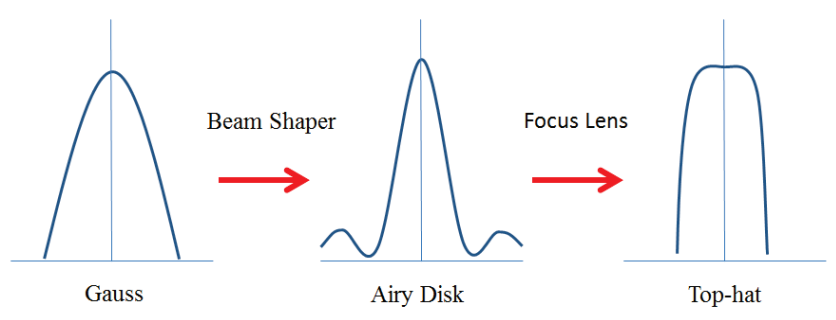


Fig. 3 The changing process of laser beam from Gaussian to Top-hat profile.

\section{Experimental setup and results}

The material used in this study is Ag/PET and the sample size is cut to $20 \times 90 \mathrm{~mm}$. The thickness of the silver foil and PET is $10 \mu \mathrm{m}$ and $300 \mu \mathrm{m}$, respectively. The silver foil is coated by the screen printing process. The laser scribing tests are performed first to observe the differences in using two different laser sources, nanosecond and picosecond laser. The laser parameters are shown in Table 1. A U-shaped cross-sectional profile of the sample can be obtained by using a nanosecond laser as shown in Fig. 4, but it has a greater heat affected zone (HAZ) as shown in Fig. 5. The HAZ is defined by the blackened region of the silver foil after scribing process as shown in Fig. 5 (A). Due to the different beam profile of the pulse durations investigated, it is easier to create a U-shaped cross-sectional profile by using a nanosecond laser. In addition, there are two kinds of material removal via laser machining: photothermal and photochemical mechanisms. In this study, for the ablation of silver thin films, both regimes, nanosecond and picosecond, are photothermal process. Only the thermal diffusion length, and then the heat affected zone, changes with the pulse duration. In the case of PET, it depends on the material properties. In addition, multiphotonic absorption could occur in the picosecond regime, and the probability to induce photochemical mechanisms in this regime is negligible.

In this work, a picosecond laser is selected as the laser sources for this study and aims to obtain a U-shaped crosssectional profile, so that it can completely replace a nanosecond laser of its advantage of the cross-sectional profile. The experiments were conducted with three different groups, control, experimental group 1, and group 2. In the control group, the experiments are performed without using any beam shaping technology, means the laser beam is in a Gaussian distribution for scribing process. In the experimental group 1 and 2, the laser beam shaping technology was used to generate the Top-hat beam profile. The major difference between the experimental group 1 and 2 are in the different dimensions of the laser beam. On the beam shaper, the magnification ring was turned to value 10 for experimental group 1 and turned to 20 for group 2. The focusing ring on the beam shaper are set to zero in both experimental groups. In experimental group 1 and 2, the measured laser beam size are approximately $6.5 \mathrm{~mm}$ and $3.6 \mathrm{~mm}$, respectively. The beam size measurements are performed at a distance of $10 \mathrm{~cm}$ after the beam shaper. In the experimental group 2, the laser beam size is reduced of around $55 \%$, relative to the experimental group 1 . All other laser parameters including pulse duration, repetition frequency, fluence, and scribing speed remain the same in these three different experimental groups.

Before the scribing experiments, a laser beam profiler is used to measure the energy distribution of the laser beam as shown in Fig. 6. A conventional Gaussian distribution and Top-hat beam are measured before and after laser beam shaping, respectively.
Table 1 Laser parameters

\begin{tabular}{|c|c|c|c|c|c|}
\hline & $\begin{array}{c}\text { Pulse } \\
\text { duration }\end{array}$ & $\begin{array}{c}\text { Repetition } \\
\text { freq. }\end{array}$ & $\begin{array}{c}\text { Pulse } \\
\text { energy }\end{array}$ & $\begin{array}{c}\text { Scribing } \\
\text { speed }\end{array}$ & $\begin{array}{c}\text { Laser } \\
\text { type }\end{array}$ \\
\hline $\begin{array}{c}\text { Pico- } \\
\text { second } \\
\text { laser }\end{array}$ & $10-15 \mathrm{ps}$ & $500 \mathrm{kHz}$ & $13 \mu \mathrm{J}$ & $100 \mathrm{~mm} / \mathrm{s}$ & $\begin{array}{c}\mathrm{MOPA} \\
532 \mathrm{~nm}\end{array}$ \\
\hline $\begin{array}{c}\text { Nano- } \\
\text { second } \\
\text { laser }\end{array}$ & $20-35 \mathrm{~ns}$ & $100 \mathrm{kHz}$ & $30 \mu \mathrm{J}$ & $100 \mathrm{~mm} / \mathrm{s}$ & $\begin{array}{c}\text { Q-switch } \\
532 \mathrm{~nm}\end{array}$ \\
\hline
\end{tabular}
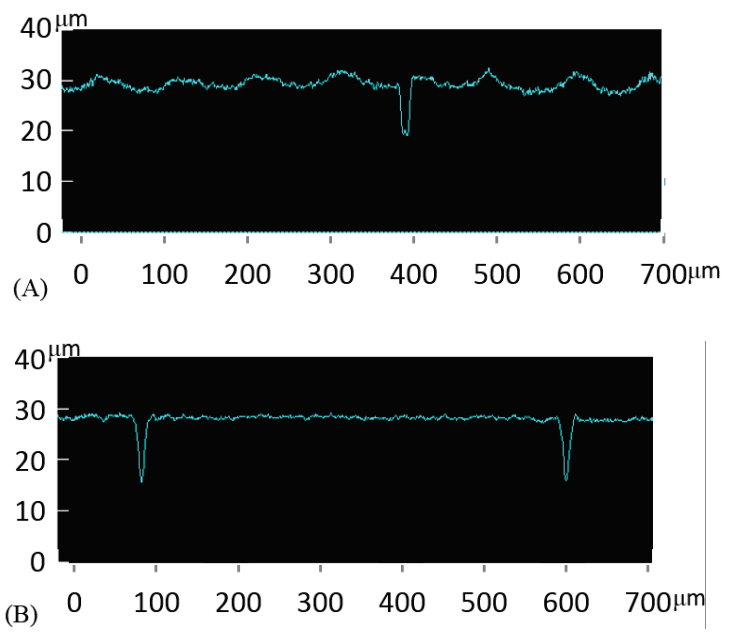

Fig. 4 Cross-sectional view of the scribed workpieces by a (A) nanosecond laser and (B) picosecond laser.
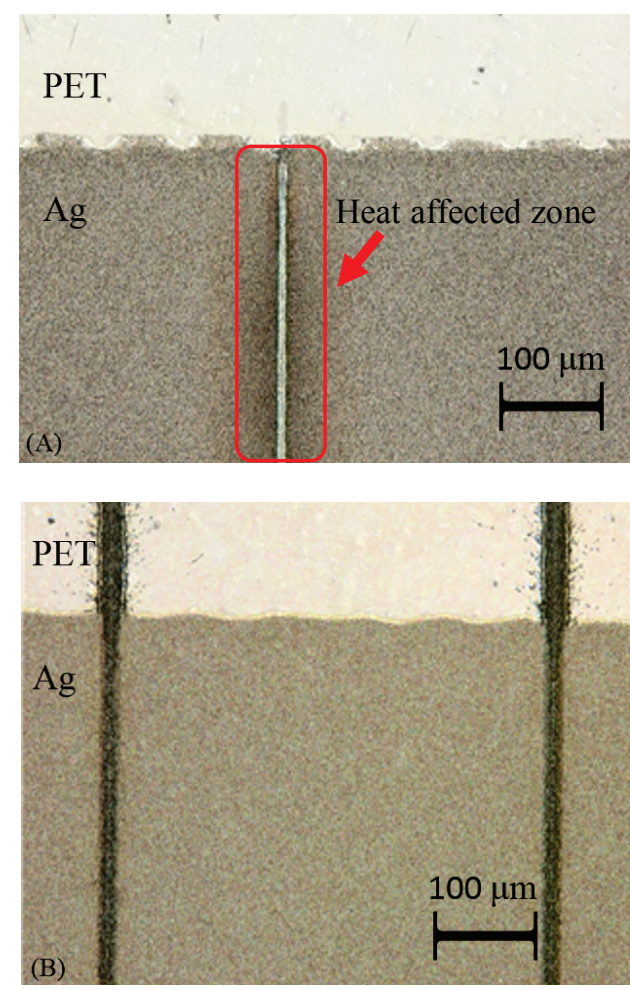

Fig. 5 Comparison of the thermal effect on scribed workpieces by a (A) nanosecond laser and (B) picosecond laser. 


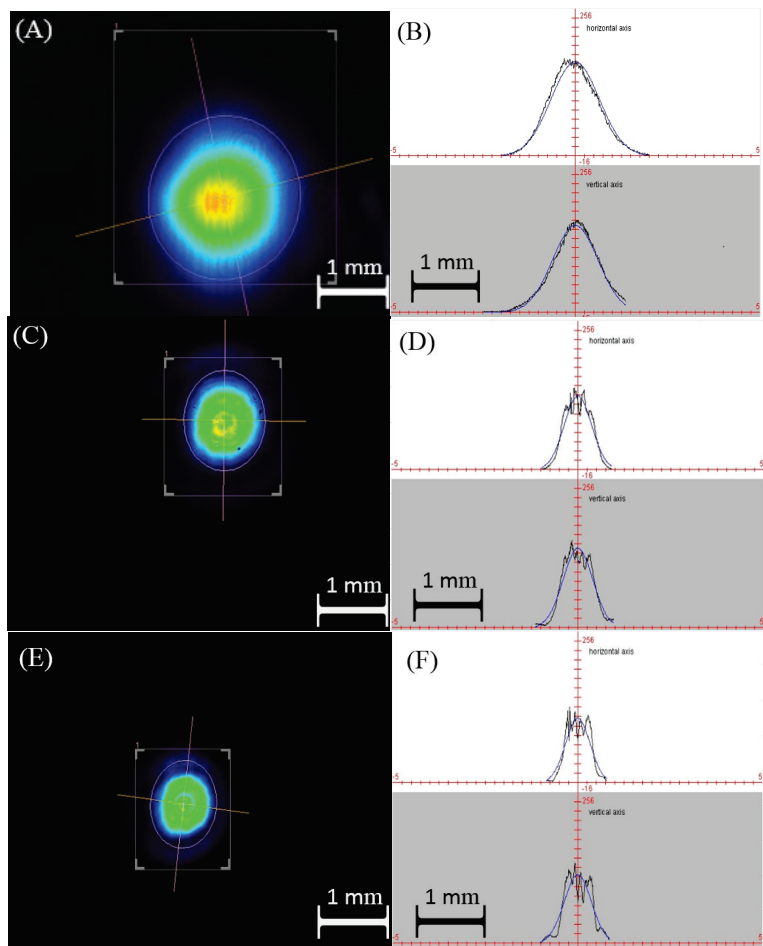

Fig. 6 Comparison of the various beam profile. Without laser beam shaping technology: Gaussian beam distribution (control group) (A) 2D intensity distribution (B) Crosssectional intensity. With laser beam shaping technology: Top-hat beam distribution (experimental group 1) (C) 2D intensity distribution (D) Cross-sectional intensity; Top-hat beam distribution (experimental group 2) (E) 2D intensity distribution (F) Cross-sectional intensity.

This study does not intend to focus on parameters optimization of laser scribing process, but mainly to create the Top-hat beam with the beam shaping technology. This optical transformation helps to reduce significantly the heat affected zone (HAZ) on the scribed workpieces, and thus, increases the productivity of material processing due to more efficient usage of the laser energy. Another typical Gaussian laser beam scribing control group is used for comparison. The features of using a Gaussian beam for scribing process is that the scribed lines are most with irregular trench edges and have a wider HAZ. However, the features of using a Top-hat beam for scribing process is that the scribed lines are with even trench edges, steep walls, and almost without HAZ as shown in Fig. 5 (B).

In the beginning of the experiments, the cross-sectional profile of scribed workpieces are first examined to confirm whether the expected V-shaped or U-shaped cross-sectional profile are observed or not. Fig. 7 shows the microscopic images of the cross-sectional profile of scribed samples. From the control group, the cross-sectional profile of the workpiece is V-shaped as shown in Fig. 7 (A). However, after beam shaping the cross-sectional profile of U-shaped is obtained from the experimental groups as shown in Fig. 7 (B) (experimental group 1) and Fig 7 (C) (experimental group 2). The experimental laser parameters are: laser frequency $500 \mathrm{kHz}$, laser pulse energy $13 \mathrm{uJ}$, scribing speed $100 \mathrm{~mm} / \mathrm{s}$ and the laser pulse spacing is $0.001 \mathrm{~mm}$. In addition, the laser focusing position is different due to the slightly difference between the spot sizes, but they are all in focus.

After the observation of the microscopic images, the scribed depth of workpiece is then measured by a Laser Scanning Confocal Microscopy (LSCM) (KEYENCE VKX200K). The reason of using LSCM for the measurements of scribed depth is that it has an advantage of high accuracy and the measurements of depth can be performed without damaging the workpiece. Fig. 8 shows the measured patterns after laser scribing by the LSCM. Fig. 8 (A) (B) (C) are the top and the measured cross-sectional views of the scribed patterns from the control group and experimental groups, respectively. In this study, the scribing experiments are performed three times with the same laser parameters to verify if the U-shaped cross-sectional profile of the workpiece can be obtained after beam shaping. Fig. 9 shows three cross-sectional profiles of the workpieces from the control group. Fig. 10 and Fig. 11 show the individual three cross-sectional profiles of the workpieces with the same laser parameters from experimental group 1 and 2, respectively. Fig. 12 is the comparison chart of the average crosssectional profiles from the control group, the experimental group 1 and 2.
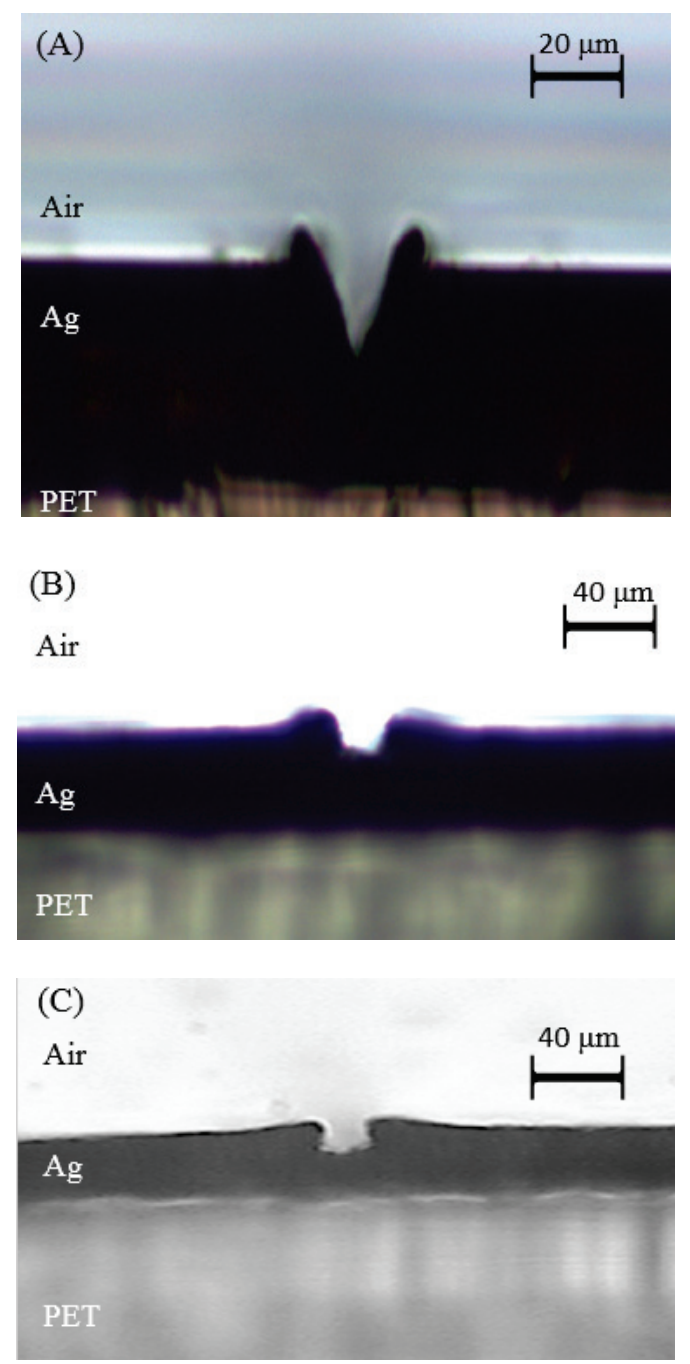

Fig. 7 Microscopic images of the cross-sectional profile of the scribed workpieces (A) control group (B) experimental group 1 (C) experimental group 2. 

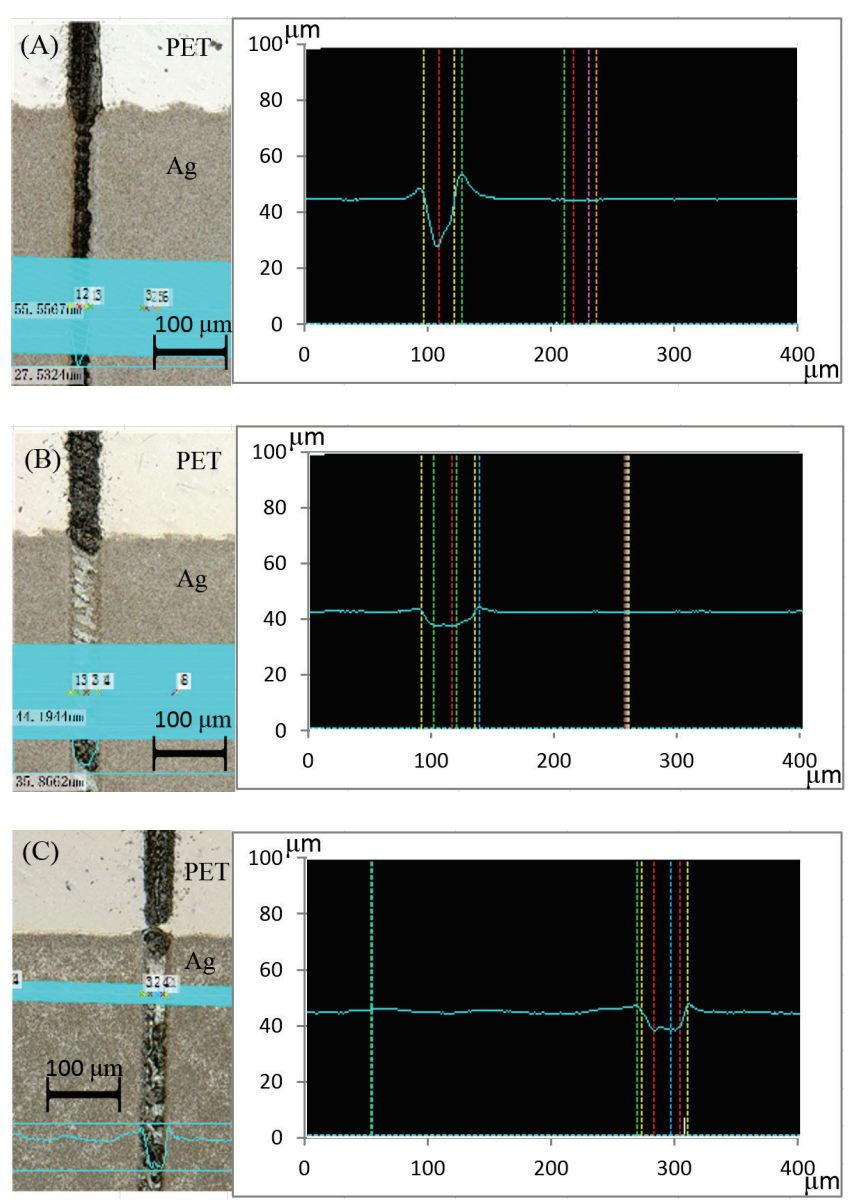

Fig. 8 Measurements of scribed depth by a Laser Scanning Confocal Microscopy (A) control group, (B) experimental group 1, (C) experimental group 2.

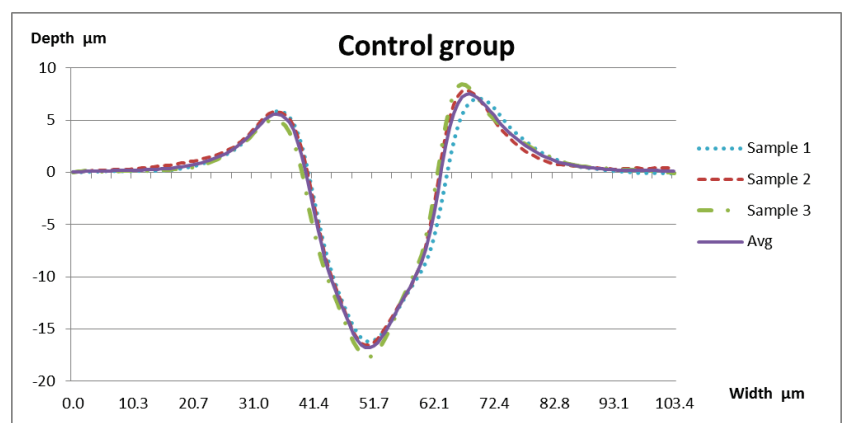

Fig. 9 Three cross-sectional profiles of the workpieces with the same laser parameters from the control group.

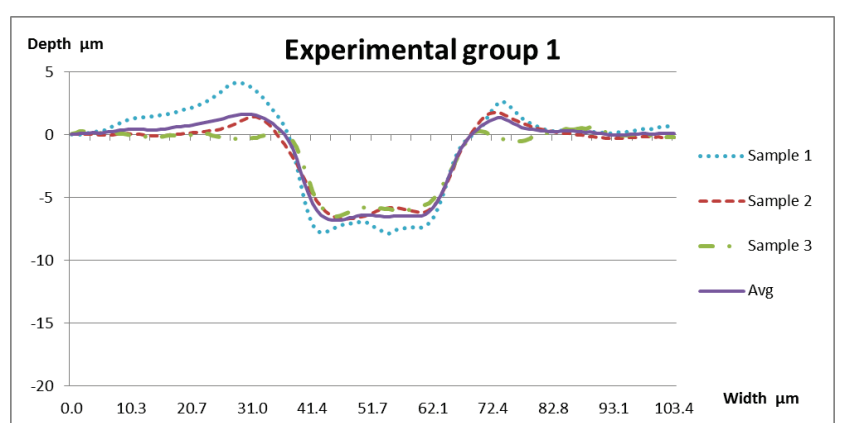

Fig. 10 Three cross-sectional profiles of the workpieces with the same laser parameters from the experimental group 1 .

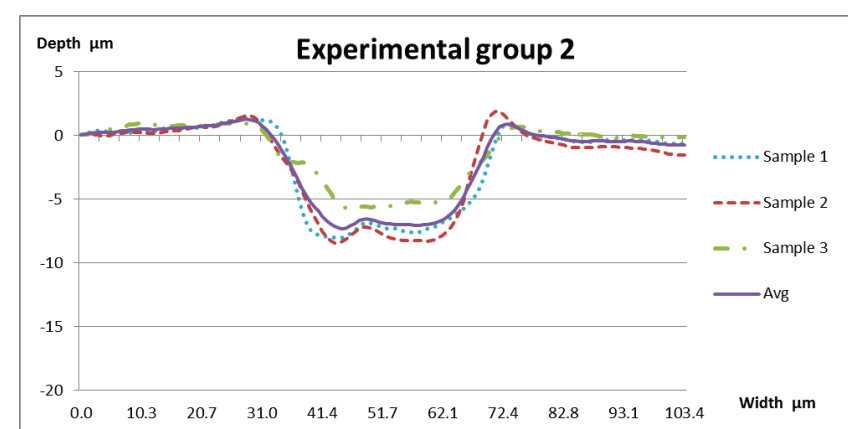

Fig. 11 Three cross-sectional profiles of the workpieces with the same laser parameters from the experimental group 2 .

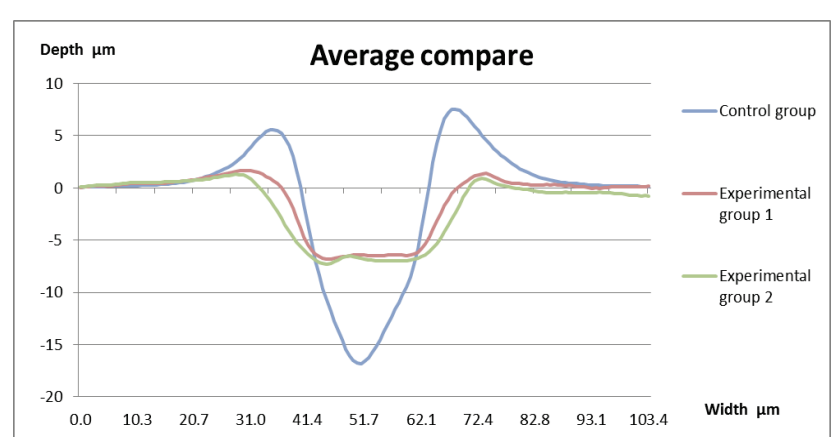

Fig. 12 Comparison chart of the average cross-sectional profiles from the control group, experimental group 1 and group 2.

\section{Discussions}

From the comparison of the various beam profiles as shown in Fig. 6, it verified that the laser beam with the Gaussian distribution was successfully converted into a Top-hat beam distribution by a beam shaper which ensured that a U-shaped cross-sectional profile of the workpiece after laser scribing process can be obtained. Moreover, after the completion of laser scribing process, the crosssectional profile of the workpiece can be observed by a microscope as shown in Fig. 7. It can be easily seen that a U-shaped cross-sectional profile is created from the experimental groups, and a V-shaped cross-sectional profile is generated from the control group. Furthermore, from the measurements of scribed depth by the LSCM, it shows that a V-shape cross-sectional profile of the workpiece from the control group generally has a deeper scribed depth and with a larger heat-affected zone (the margin of scribed patterns has a higher hill). However, after beam shaping, a Ushaped cross-sectional profile is obtained in the experimental groups, which can effectively reduce the scribing depth to avoid the damages of the substrate, PET. In addition, the laser scribed linewidth also increases, and the thermal effect is smaller, compared to the control group.

Chen et al. [6] reported on using a laser beam shaper component to obtain a Top-hat beam to perform electrode patterning on ITO thin films on the glass or plastic substrate. Furthermore, they also developed a laser annealing system for heat treatment on the ITO thin films with the use of a uniform laser beam profile and with a proper laser intensity [7]. In addition, Shang et al. [8] investigated the 
curing process under different laser intensity profiles obtained with the beam shaping device.

Compared with other works, we have successfully changed the original V-shaped cross-sectional profile of workpieces into a U-shaped profile on the Ag/PET substrate by using a picosecond laser with a beam shaper, which completely replaces a nanosecond laser of its advantage of the U-shaped cross-sectional profile. In addition, in the beam shaping setup, two different beam shaping parameters were used to obtain different beam sizes for scribing process. From our results, it shows that the PET substrate is less damaged with the U-shaped groove, compared to the V-shaped groove. Moreover, the scribed samples are almost without HAZ because after beam shaping process, a uniform laser intensity profile is obtained for scribing.

In this study, the same laser parameters are performed three times and their averages are calculated as well. Fig. 9 to 12 shows the average cross-sectional profiles of the laser scribing experiments, and it is verified that the repeatability of our experiments are acceptable. In addition, ten scribed samples were measured by a multimeter (Fluke, Model $115)$ to check if both sides of the thin film showed an open loop (OL) state after laser scribing process with the proper laser parameters. The measured results show that the isolation on the ten silver conductive thin films can be successfully achieved. Moreover, from the results showing in figure 12 , the geometry of U-shape profiles of the scribed lines are similar and thus, the estimated dielectric strength of each scribed line should have no big differences. Meanwhile, in the beam shaping setup, two different beam shaping parameters were used to obtain different beam sizes (6.5 $\mathrm{mm}$ and $3.6 \mathrm{~mm}$, referrer to group 1 and 2 parameters) for the scribing process. From our results (figure 12), it indicates that the cross-sectional profile of the scribed lines with different parameters of group 1 or 2 are all U-shape and the there is no significant differences in geometry either. Possible reason may be after the beam shaping, different beam sizes do not have a significant impact on the scribed profile since all other laser parameters are remaining the same.

\section{Conclusions}

In this study, we explore the differences between the nanosecond and picosecond laser in laser scribing process. The results indicate that the heat-affected zone of the workpiece using a nanosecond laser is greater than using a picosecond laser due to the different beam profile of the pulse durations investigated. Laser scribing using a nanosecond laser is one kind of photothermal mechanism, and thus a Ushaped cross-sectional profile of the workpiece is easily created. In photothermal mechanism, the energy of the laser beam is absorbed by the material first and converted into heat to make the material melt. Due to the different beam profile of the pulse durations, a nanosecond laser and a picosecond laser can easily produce a U-shaped and a Vshaped cross-sectional profile, respectively.

This study explores whether a beam shaper can convert a Gaussian beam into a Top-hat beam. The experiments were divided into two experimental and one control groups for laser scribing processes and then implemented three times with the same laser scribing parameters for experimental validation. This study successfully uses a picosecond laser with a beam shaper to change the original V-shaped cross-sectional profile of the workpiece into a U-shaped cross-sectional profile. Therefore, it increases another feature of using a picosecond laser in scribing process and the conclusions can be summarized as follows:

1. The workpiece has a smaller heat-affected zone without a blackened area on it.

2. Due to the small heat-affected zone and short pulse duration, the edge of the workpiece after laser scribing has a better quality and therefore improves the machining accuracy.

3. A picosecond laser for scribing process was successfully performed to produce a U-shaped crosssectional profile of the workpiece and thus reduces the damage of substrate PET.

This study demonstrates that a picosecond laser with a beam shaper allows a Gaussian beam to be converted into a Top-hat beam, extending the use of laser processing. In addition, the laser scribing parameters in this study have not been optimized. In the future work, if the laser parameters could be optimized by the methods of Grey relational theory or Taguchi [13], the quality of laser scribing can be further improved.

\section{References}

[1] M.K.M. Ali, K. Ibrahim, and E.M. M kawi: Materials Science in Semiconductor Processing, 16, (2013) 593.

[2] B.I. Noh, J.W. Yoon, K.S. Kim, S. Kang, and S.B. Jung: Microelectronic Engineering, 103, (2013) 1.

[3] S. Venkat, and C. Dunsky, Proc. SPIE, Photon Processing in Microelectronics and Photonics V: San Jose, (2006) p. 610602.

[4] G. Raciukaitis, M. Brikas, M. Gedvilas, and T. Rakickas: Applied Surface Science, 253, (2007) 6570.

[5] M. Park, B.H. Chon, H.S. Kim, S.C. Jeoung, D. Kimb, J.I. Lee, H.Y. Chu, and H.R. Kim: Optics and Lasers in Engineering, 44, (2006) 138.

[6] M.F. Chen, W.T. Hsiao, Y.S. Ho, S.F. Tseng, and Y.P. Chen: Thin Solid Films, 518, (2009) 1072.

[7] M.F. Chen, K.M. Lin, and Y.S. Ho: Optics and Lasers in Engineering, 50, (2012) 491.

[8] S. Shang, D. Wellburn, E. Fearon, S. Yan, S. Edwardson, G. Dearden, and K.G. Watkins: Optics and Lasers in Engineering, 51, (2013) 527.

[9] A.V. Laskin: "How effective is using of laser beam?", www.mt-berlin.com.

[10]F.M. Dickey, S.C. Holswade, and D.L. Shealy: "Laser Beam Shaping Applications" Published in 2006 by CRC Press Taylor \& Francis Group.

[11]D. Sheal: "Laser Beam Shaping-Theory and Techniques" ed. by F.M. Dickey and S.C. Holswade (New York: Marcel Dekker, 2000), Chap. 4.

[12] J. J. Camacho, L. Díaz, M. Santos, L. J. Juan, and J. M. L. Poyato: "Laser Beams: Theory, Properties and Applications", (New York: Nova Science Publishers, 2011, Inc.), P.419.

[13]H.C. Chuang, and W.F. Lee: Optics and Laser Technology, 57, (2014) 149.

(Received: January 1, 2014, Accepted: June 27, 2014) 